\title{
LA EVALUACIÓN EN LAS CLASES DE QUÍMICA DESDE EL ENFOQUE DE ENSEÑANZA PARA LA COMPRENSIÓN ${ }^{1}$
}

Adny Vásquez ${ }^{2}$ lore12 81@hotmail.com, Julieth Orobio ${ }^{2}$ yulipianis@hotmail.com

\section{Resumen}

En el presente escrito se aborda la importancia de la evaluación de aprendizajes en un contexto educativo, desde el enfoque de Enseñanza para la Comprensión (EpC). Para tal fin, durante el primer semestre del año 2012 se realiza la observación y el registro de clases en un diario de campo y se aplica un instrumento (encuesta) a los estudiantes de grado décimo del Colegio Champagnat, con el que se pretende conocer sus percepciones sobre la evaluación, ¿qué entienden por evaluación?, ¿de qué manera se les evalúa? y ¿cómo les gustaría ser evaluados en las clases de Química?

Con base en los resultados obtenidos y las observaciones realizadas, se logran identificar algunas concepciones de los estudiantes sobre la evaluación educativa y en particular la llevada a cabo en las clases de Química. Debido a esto, en el contexto de la práctica II, se plantean fortalecer aspectos que son de gran importancia en el proceso de evaluación de los aprendizajes en las clases de química y trabajar para superar las debilidades identificadas en esta fase preliminar del estudio.

\section{Palabras Clave}

Enseñanza para la Comprensión, Enseñanza de la Química, Actitudes de los estudiantes, Evaluación, Reacciones Químicas.

\footnotetext{
${ }^{1}$ Trabajo desarrollado en el colegio Champagnat. Práctica Pedagógica y Didáctica I

${ }^{2}$ Estudiantes del Departamento de Química de la UPN
} 


\section{INTRODUCCIÓN}

No se puede desconocer que la naturaleza de la evaluación gira entorno al concepto de valor, de cualquier forma, todos los métodos, técnicas y procedimientos vinculados a la evaluación no han tenido otro propósito que establecer normas y principios que permitan evaluar, valorar, estimar, tasar o medir acciones, trabajos, respuestas o actitudes. (Cerda, $\mathrm{H}$. 2000). A través del tiempo el concepto de evaluación ha cambiado, ésta ya no es simplemente un instrumento que mide los conocimientos adquiridos por el estudiante, sino que se constituye en un proceso que acompaña la formación del educando (Santos, M. 2002).

El Colegio Champagnat de Bogotá en su PEI, "Champagnat ama la tierra", incluye la evaluación como uno de los tópicos pilares de la institución educativa. Precisamente, en este campo, la institución ha venido pasando por un proceso de transformación lento. Se pasó de la mera calificación cuantitativa de tareas, a la información descriptiva a través de desempeños involucrados en la formación de competencias propias de las disciplinas, pues el modelo se sustenta en el enfoque de Enseñanza para la Comprensión; este enfoque involucra a la evaluación diagnóstica continua, proceso que se debe realizar permanentemente.
El marco conceptual de la EpC hace evidente la necesidad que la evaluación vaya más allá de un examen cuantitativo al final de cada unidad o del curso. Los estudiantes precisan de oportunidades para reflexionar sobre sus desempeños durante el aprendizaje de nuevos conceptos o habilidades cognitivas y no sólo al final de este aprendizaje. Estas reflexiones deben realizarse teniendo en cuenta las metas de comprensión planteadas y siguiendo los criterios e información brindados por el docente.

\section{DESCRIPCIÓN DEL PROBLEMA}

Dado que para muchos educadores y educandos la evaluación es un mecanismo de medición, caracterizado por un juicio de valor, se hace necesario conocer las concepciones de los estudiantes acerca de la evaluación de aprendizajes, para que de esta manera se puedan identificar las fortalezas y debilidades que permitan favorecer el proceso de enseñanzaaprendizaje de la Química.

Por esto, en el marco de este trabajo surge la siguiente pregunta:

¿Qué concepciones tienen los estudiantes de grado décimo del Colegio Champagnat acerca de la evaluación en las clases de Química? 


\section{MARCO TEÓRICO}

Una definición importante que se viene dando desde hace mucho tiempo afirma que, "la evaluación es el enjuiciamiento sistemático de la valía o el mérito de un objeto" (Stuffleabeam, D 1987). Esta definición se centra en el término valor e implica que la evaluación siempre supone juicio.

Anteriormente la evaluación solo se utilizaba como una forma de medir o cuantificar, era seguida por un régimen positivista y conductista pues se comportaba como una instancia de control lo mismo que los exámenes. Con los años, la evaluación dejó de ser un instrumento de control para transformarse en una herramienta vinculada con la problematización de los valores y el sentido de estos en la situación observada. El valor de la evaluación solo reside en la utilidad de la información que aporta para la toma de decisiones. La evaluación más que un instrumento de medir o producir conocimientos, es fundamentalmente una experiencia investigativa (Cerda, H. 2000).

Por otra parte, la Enseñanza para la Comprensión involucra a los educandos en cuatro aspectos: un tópico generativo, desempeños de comprensión, metas de comprensión y la evaluación diagnóstica continua. Los desempeños de comprensión exigen que los estudiantes apliquen, amplíen y sinteticen lo que saben, además controlan y promueven el avance de los educandos por medio de evaluaciones diagnósticas continuas con criterios directamente vinculados con las metas de comprensión. (Stone, M.1999)

La evaluación diagnóstica continua, según Martínez (2007, p 30), "se basa en criterios públicos vinculados con metas de comprensión, tienen lugar a menudo, son hechas por los alumnos, y los docentes por igual y configuran la planificación. A la vez que estiman el progreso de los alumnos". Para encontrarle sentido a lo aprendido, es necesario que los estudiantes reciban retroalimentación sobre sus desempeños de comprensión; por tal motivo, la evaluación debe ser propuesta con base en los desempeños de comprensión.

En el contexto de la evaluación participan los cuatro niveles básicos de cualquier investigación: descripción, clasificación, explicación y comprensión. Por tanto, se ve la evaluación como una función que reorienta el aprendizaje, corrige errores, modifica las actividades, acelera el ritmo, identifica los problemas y las necesidades, pero a su vez potencia y consolida lo positivo. (Cerda, $\mathrm{H}$. 2000)

\section{OBJETIVOS}

\section{General}

$\checkmark \quad$ Identificar las concepciones que tienen los estudiantes de grado décimo del Colegio Champagnat sobre la evaluación de aprendizajes en las clases de Química, con el fin de elaborar una propuesta de trabajo que permita abordar las debilidades y fortalezas detectadas.

\section{Específicos}

$\checkmark \quad$ Observar sistemáticamente las clases de Química en grado décimo prestando especial atención a los aspectos evaluativos desde la perspectiva del enfoque EpC.

$\checkmark \quad$ Diseñar y aplicar un instrumento de para identificar algunas concepciones de los estudiantes sobre la evaluación, estableciendo categorías para el procesa- 


\section{METODOLOGÍA}

La metodología utilizada para el desarrollo de este trabajo, se puede sintetizar de la siguiente forma

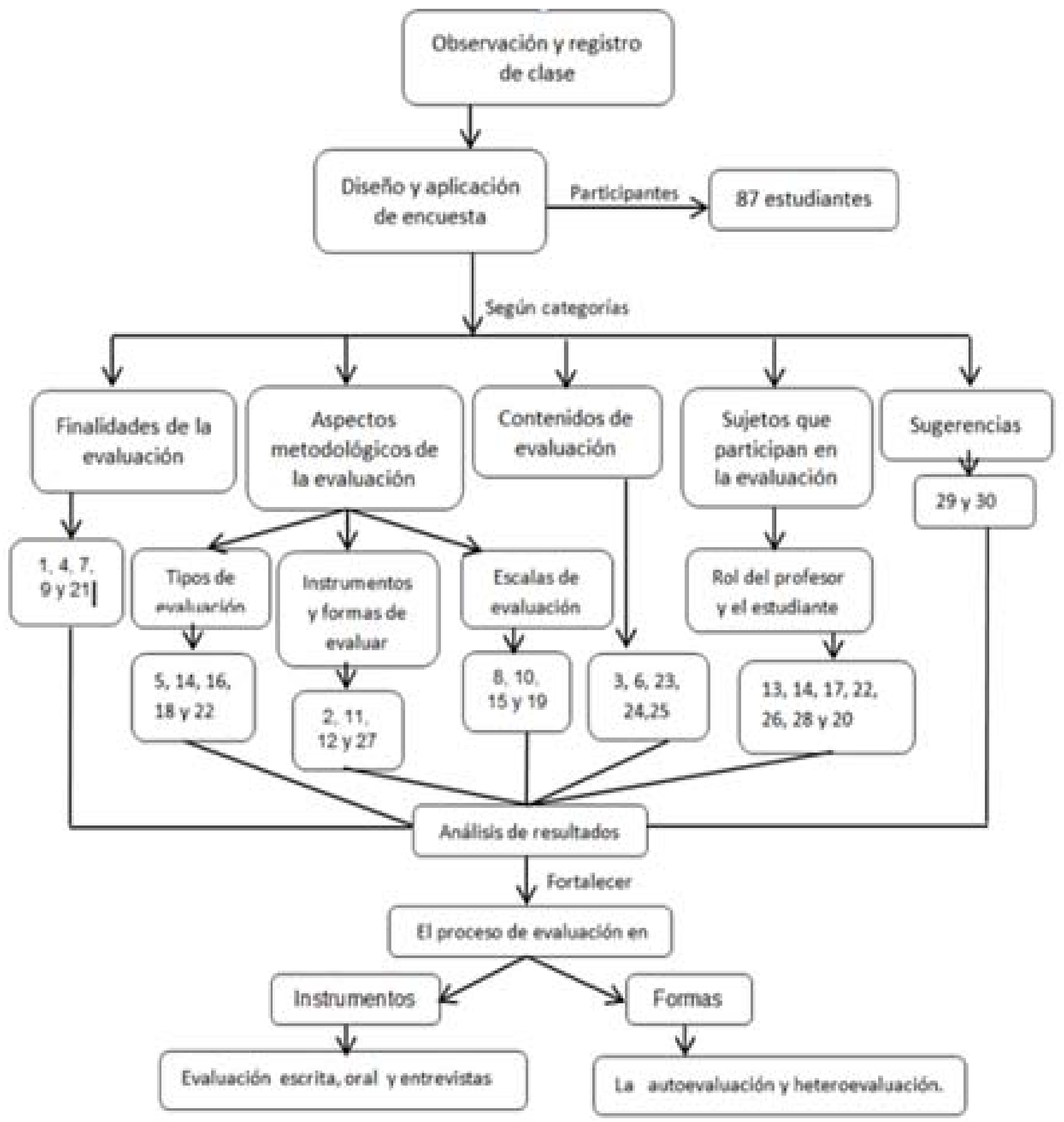

Figura 1. Síntesis del proceso 


\section{METODOLOGÍA}

\section{Descripción del instrumento}

La observación sistemática de las clases de Química y el instrumento aplicado (encuesta), permitieron identificar las opiniones de los estudiantes respecto a la evaluación.

El instrumento comprende 30 ítems. Del número uno al 28 , se plantean afirmaciones utilizando una escala Likert, considerando las siguientes opciones de respuesta:

1. Totalmente de acuerdo

2. De acuerdo

3. Indeciso

4. En desacuerdo

5. Totalmente en desacuerdo

Los ítems números 29 y 30 corresponden a preguntas abiertas en relación con posibles sugerencias de los estudiantes frente a su proceso evaluativo. (Anexo).
Para el análisis de la información, las afirmaciones de la encuesta se agruparon en las siguientes categorías y subcategorías:

Finalidades de la evaluación

Afirmaciones 1, 4, 7, 9, 21

Aspectos metodológicos de la evaluación

Tipos

Afirmaciones 5, 14, 16, 18,22

Escalas

Afirmaciones 8, 10, 15, 9

Instrumentos y formas

Afirmaciones 2, 11, 12 , 27

Contenidos

Afirmaciones 3, 6, 23, 24, 25

Sujetos que participan en la evaluación

Afirmaciones 13, 14, 17, 20,

$22,26,28$ 


\section{RESULTADOS Y ANÁLISIS}

En la siguiente tabla, se presentan los resultados de la encuesta (ítems 1 a 28). Se advierte que para algunos de los ítems no todos los estudiantes no marcaron ninguna respuesta.

\begin{tabular}{|c|c|c|c|c|c|c|}
\hline Afirmaciones & 1 & 2 & 3 & 4 & 5 & total \\
\hline 1 & 9 & 41 & 18 & 15 & 2 & 85 \\
\hline 2 & 6 & 15 & 33 & 20 & 12 & 86 \\
\hline 3 & 10 & 32 & 29 & 12 & 3 & 86 \\
\hline 4 & 18 & 54 & 12 & 2 & 1 & 87 \\
\hline 5 & 6 & 7 & 17 & 39 & 18 & 87 \\
\hline 6 & 3 & 25 & 33 & 16 & 10 & 87 \\
\hline 7 & 7 & 37 & 27 & 11 & 5 & 87 \\
\hline 8 & 24 & 29 & 19 & 12 & 3 & 87 \\
\hline 9 & 17 & 41 & 26 & 3 & 0 & 87 \\
\hline 10 & 25 & 35 & 14 & 8 & 5 & 87 \\
\hline 11 & 14 & 20 & 31 & 12 & 10 & 87 \\
\hline 12 & 5 & 11 & 34 & 23 & 13 & 86 \\
\hline 13 & 5 & 35 & 24 & 16 & 4 & 84 \\
\hline 14 & 9 & 39 & 25 & 9 & 4 & 86 \\
\hline 15 & 4 & 27 & 23 & 23 & 10 & 87 \\
\hline 16 & 1 & 16 & 27 & 24 & 19 & 87 \\
\hline 17 & 7 & 18 & 21 & 20 & 21 & 87 \\
\hline 18 & 10 & 23 & 32 & 16 & 6 & 87 \\
\hline 19 & 25 & 36 & 18 & 8 & 0 & 87 \\
\hline 20 & 13 & 31 & 34 & 7 & 2 & 87 \\
\hline 21 & 7 & 36 & 28 & 12 & 3 & 86 \\
\hline 22 & 6 & 17 & 24 & 28 & 12 & 87 \\
\hline 23 & 19 & 46 & 17 & 4 & 1 & 87 \\
\hline 24 & 16 & 45 & 14 & 8 & 3 & 86 \\
\hline 25 & 40 & 29 & 12 & 5 & 1 & 87 \\
\hline 26 & 38 & 28 & 12 & 7 & 2 & 87 \\
\hline 27 & 16 & 40 & 23 & 7 & 1 & 87 \\
\hline 28 & 2 & 20 & 14 & 28 & 23 & 87 \\
\hline
\end{tabular}

Tabla 1. Concepciones de los estudiantes sobre la evaluación de aprendizajes 
A continuación se presenta un histograma en el cual se graficaron las respuestas a las afirmaciones 1 a 28, relacionando el grado de acuerdo y desacuerdo, para ello el resultado de totalmente de acuerdo es sumado con el de acuerdo y el totalmente en desacuerdo con el desacuerdo; este se muestra en la figura 2.
La finalidad del trabajo del evaluador es orientar la selección de informaciones y puntos de vista que permitan a los estudiantes ampliar la comprensión, la reflexión, y a su vez tener un espacio de dialogo y negociación entre profesor-alumno para la discusión del sentido y naturaleza de las finalidades de la evaluación,

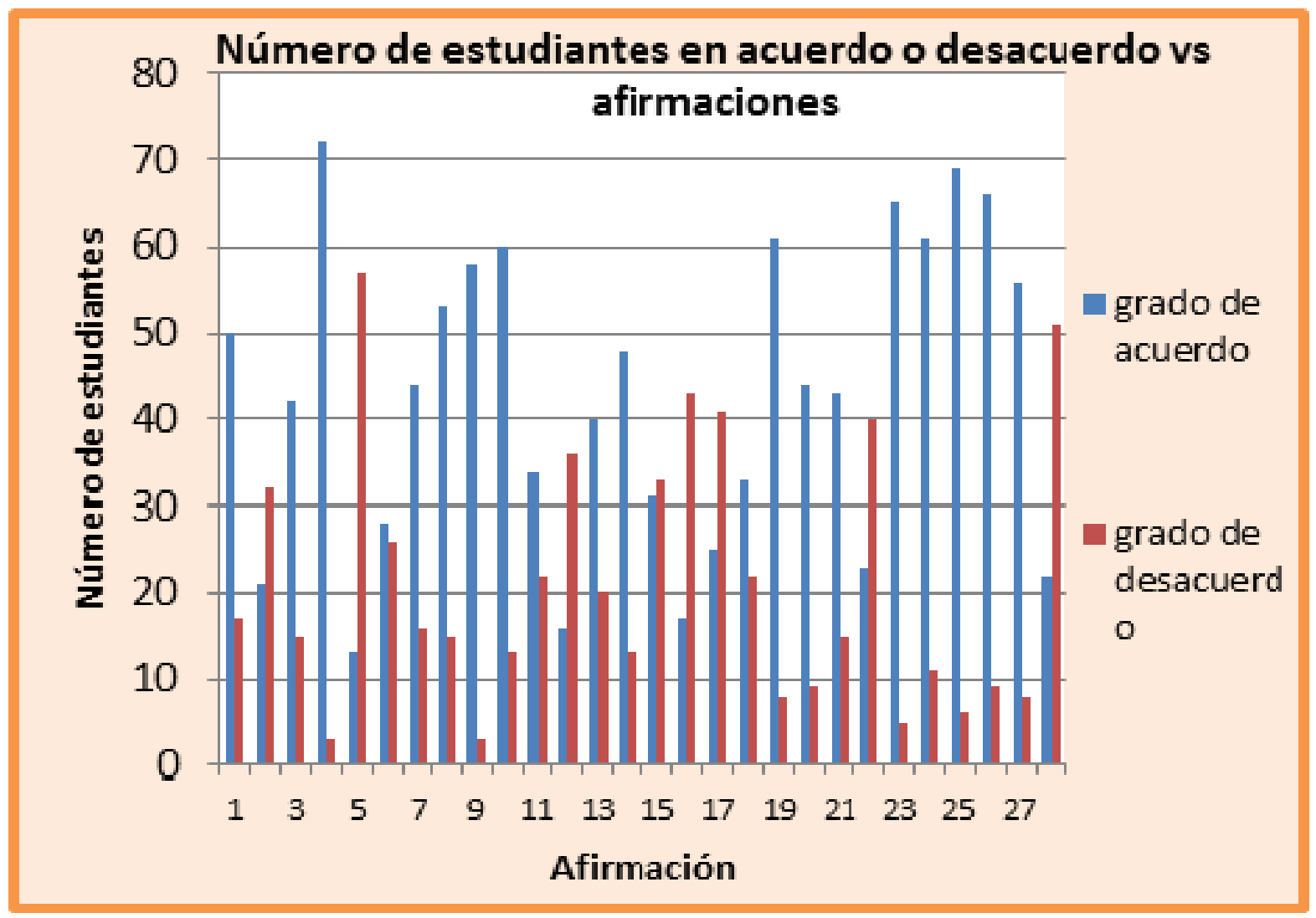

Figura 2. Grado de acuerdo y desacuerdo de los estudiantes respecto a las afirmaciones de la encuesta

Se observa que la mayoría de las afirmaciones muestran una tendencia a estar más de acuerdo que en desacuerdo a excepción de las afirmaciones 15, 16, 17, 22 28.

Respecto a la categoría finalidades de la evaluación, las respuestas de los estudiantes en general fueron positivas mostrando tendencias a favor de cada afirmación. además, dar lugar a la valoración de los puntos de vista del programa y los métodos que facilitan la realización de esos juicios. (Santos, M. 1995)

La evaluación puede servir para muchas finalidades como aprender, diagnosticar, valorar, comprender, reorientar, mejorar, motivar y reflexionar. (Santos, M. 2002). 
En cuanto a la categoría aspectos metodológicos de la evaluación y la subcategoría tipos de evaluación, se encontró que la mayoría de la población está en desacuerdo con la afirmación cinco, ya que 57 estudiantes ven la evaluación como un proceso, y ratifican la tendencia de la afirmación uno (Anexo)

Al indagar acerca de los procesos de autoevaluación, coevaluación y heteroevaluación, se evidencia que estos no se han llevado a cado en las clases de química. La mayoría de la población está en desacuerdo con las afirmaciones asociadas a estos aspectos.

Para la subcategoría instrumentos y formas de evaluación, se tiene que existen dos tipos de pruebas: las objetivas y las subjetivas. Las primeras son construidas con base en reactivos cerrados y específicos, de modo que sus respuestas son procesadas de acuerdo con criterios estadísticos y matemáticos. En cambio, las pruebas subjetivas hacen uso de preguntas abiertas o reactivos de cierta amplitud; las respuestas se construyen libremente, sin restricciones de extensión o forma (test de multiítem, de múltiple respuesta, pruebas de ensayo, entre otras.). (Cerda, H. 2000). Las respuestas de los estudiantes permiten evidenciar variedad de preferencias respecto a las formas de cómo prefieren ser evaluados. Se evidencia que un gran porcentaje de estudiantes no saben que tipo de evaluación prefieren. Por otro lado un $42 \%$ se siente bien con las evaluaciones orales pero un $39 \%$ de los estudiantes se siente bien con las evaluaciones escritas.

En cuanto a las escalas de evaluación, las respuestas a las afirmaciones 8, 10 y 19 permiten evidenciar como los estudiantes asocian una nota "valor" con la comprensión. Es muy bajo el porcentaje de estudiantes que consideran que la nota no influye en su conocimiento, o en lo que él.
No es un secreto que para muchos estudiantes lo más importante es la nota y no lo que han aprendido; ellos y sus padres se sienten gratificados con un sobresaliente (8/10 puntos) y mucho más con un excelente (10/10 puntos); sin embargo ¿qué significado tiene esta valoración si realmente ésta no define qué se comprendió?. "La asignación de números de una manera mecánica, como es común en los procedimientos cuantitativos, no garantiza la objetividad" (Santos, M. 1995, pág. 21).

La categoría contenidos de la evaluación permite reflexionar sobre el proceso de enseñanza aprendizaje el cual se realiza sobre un cuerpo de conocimientos, dado que no se puede aprender en el vacío. No se puede entonces rechazar el aprendizaje de contenidos porque son necesarios para articular el pensamiento, para adaptarse a la realidad y para poder manejarla. (Santos, M. 1995, pág. 18).

Es importante también considerar que limitar la evaluación a los contenidos supone un reduccionismo al aprendizaje del estudiante, dado que así como son importantes los contenidos lo más relevante es cómo el estudiante hace uso de esos nuevos conocimientos para emplearlos en nuevas situaciones.

Las respuestas de los estudiantes en torno a esta categoría muestran que hay relación entre los contenidos enseñados en la clase de química con los evaluados (afirmaciones 6, 23 y 24). Según la afirmación 25 , el $79 \%$ de la población considera importante los contenidos de evaluación para su proceso de aprendizaje; además se observa en los resultados de la afirmación 3, que la mayoría de los estudiantes están de acuerdo con la importancia de los conceptos evaluados. 
La categoría sujetos que participan en la evaluación permite analizar la importancia del contexto educativo y sus participantes en el proceso de evaluación tales como el estudiante, la familia, el establecimiento educativo, el medio ambiente, el medio social, los programas y los planes educativos.

Las respuestas a las afirmaciones 26,20 , 22 y 14 las cuales involucran el papel del estudiante, son positivas, pues los jóvenes reconocen cual es su función en el proceso de evaluación.

Por otro lado se muestra inconformidad en cuanto a las respuestas dadas a la afirmación 28, el papel del profesor. La mayoría de los estudiantes afirman que el profesor no da a conocer con anterioridad los porcentajes de cada trabajo o actividad que se realiza; esto también se evidencia posteriormente ya que es una de las sugerencias que hacen los estudiantes en la pregunta 30.

Este es uno de los aspectos a fortalecer en el proceso de evaluación, así como favorecer la autoevaluación y la coevaluación, dado que en el proceso de evaluación debe existir una relación profesor-estudiante estrecha.

En cuanto a las preguntas abiertas, 29 y 30 donde se les pide a los estudiantes que resalten aspectos positivos de la clase de química y que sugieran ideas de cómo les gustaría que los evaluaran, se obtuvieron los siguientes resultados:

La mayoría de estudiantes resalta la importancia de las prácticas de laboratorio, como una herramienta que les permite comprender los temas con mayor facilidad. Asimismo los talleres les permiten reforzar los temas que se tratan en las evaluaciones escritas, también se menciona la cartilla como un buen elemento de aprendizaje y evaluación, y resaltan que en la calificación de trabajos y evaluaciones el profesor les se- ñala los errores cometidos y se hacen observaciones de los aspectos en los que el estudiante está fallando para no volver a cometerlos.

En cuanto a las sugerencias algunos estudiantes proponen que sería bueno hacer evaluaciones sobre las prácticas experimentales, realizar más evaluaciones orales, que las evaluaciones sean más lúdicas, menos exámenes y más talleres, que se tenga en cuenta la opinión del estudiante, establecer fechas de las evaluaciones y porcentajes de las mismas, realizar más experimentos y que se realicen entrevistas donde el estudiante pueda demostrar lo que verdaderamente piensa y sabe.

\section{CONCLUSIONES}

Los tipos de evaluación que se llevan a cabo en el proceso de enseñanza aprendizaje en el colegio Champagnat de Bogotá, en la asignatura de química, para grado décimo, en el segundo periodo del año 2012 son: la evaluación cualitativa, diagnóstica, sumativa, parcial, formativa, y cuantitativa.

La evaluación que se lleva a cabo en el colegio Champagnat en la clase de química se encuentra bajo el marco del modelo de $\mathrm{EpC}$, dado que ésta es vista como un proceso continuo.

Los aspectos que se deben fortalecer en el proceso de evaluación hacen referencia al uso de instrumentos como la evaluación oral y entrevistas. En cuanto a las formas de evaluar es importante tener en cuenta la autoevaluación, la coevaluación y la heteroevaluación.

El colegio Champagnat cuenta con aspectos importantes en cuanto a los instrumentos de evaluación, como las cartillas y los multitalleres que incluyen las prácticas de laboratorio. 
La evaluación desde el enfoque EpC, como proceso continuo y permanente, permite concluir si los estudiantes desarrollan los desempeños y metas de comprensión propuestos.

Una de las fortalezas en el momento de la evaluación de contenidos y procedimientos, es la de las prácticas de laboratorio, las más resaltadas por los estudiantes.

Para los estudiantes, realizar prácticas de laboratorio, en cada tema, es estimulante para su motivación y gusto por la química, además de ayudarles a asimilar de mejor manera el conocimiento.

La evaluación del desempeño pretende dar a conocer a la persona evaluada, cuáles son sus aspectos fuertes o sus fortalezas y cuáles son los aspectos que requieren un plan de mejoramiento o acciones enfocadas hacia el crecimiento y desarrollo continuo, tanto personal como profesional, para impactar sus resultados de forma positiva.

\section{PROYECCIONES}

Dados los resultados obtenidos en esta fase de la práctica pedagógica y didáctica I, se proyecta continuar estructurando una propuesta de trabajo para la práctica II teniendo como eje central el problema de la evaluación de aprendizajes en las clases de Química.

\section{BIBLIOGRAFÍA}

Cerda, H. (2000). La evaluación como experiencia total logros objetivos procesos competencias y desempeño. Colombia: Magisterio

Martínez, J. (2007). La enseñanza para la comprensión. Una aplicación en el aula. Universidad Pedagógica Nacional. Bogotá.

Santos, M. (1995).La evaluación: un proceso de dialogo, comprensión y mejora. Granada: Aljibe, S.L.

Santos, M. (2002). Una flecha en la diana. La evaluación como aprendizaje. Andalucía educativa. $N^{\circ} 34$

Stuffleabeam, D. (1987). Evaluación sistemática. Guía teórica y práctica. Barcelona. Paidós

Stone, M. (1999). La enseñanza para la comprensión. Vinculación entre la Investigación y la práctica. Barcelona. Editorial Paidós 
ANEXO

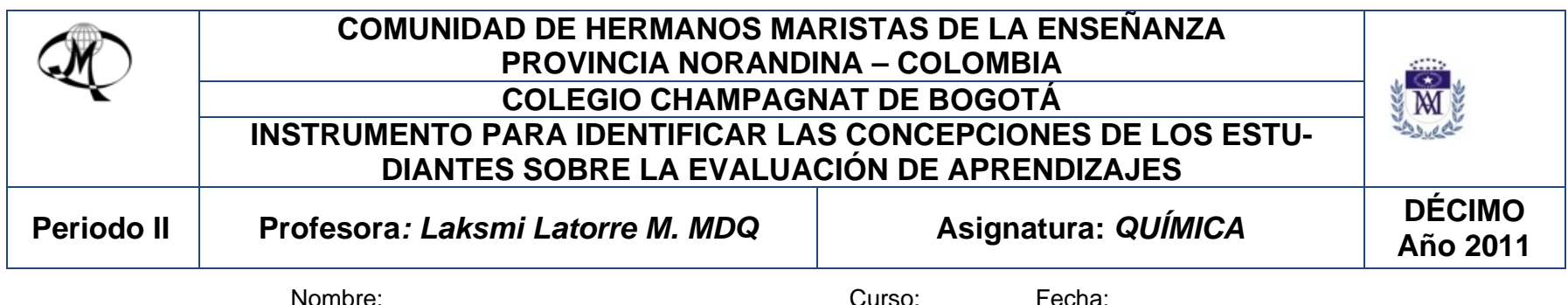

Responda las siguientes afirmaciones de acuerdo con su grado de acuerdo o desacuerdo. Marque con una $\mathrm{X}$ según la siguiente escala

1. Totalmente de acuerdo 2. De acuerdo 3. Indeciso, 4. En desacuerdo 5. Totalmente en desacuerdo

\begin{tabular}{|c|c|c|c|c|c|}
\hline & AFIRMACIONES & 1 & 2 & 3 & 5 \\
\hline 1 & La evaluación es un proceso en el cual son valorados todos los esfuerzos que se realizan durante el año. & & & & \\
\hline 2 & Me va mejor cuando las preguntas del examen son memorísticas & & & & \\
\hline 3 & Los conceptos que se valora en el examen tiene importancia para mi vida. & & & & \\
\hline 4 & La evaluación me permite reflexionar sobre mi proceso de aprendizaje. & & & & \\
\hline 5 & La forma en la que me evalúan solo tiene en cuenta un examen final & & & & \\
\hline 6 & Con los conceptos aprendidos puedo dar respuesta a problemas de la sociedad o de la cotidianidad & & & & \\
\hline 7 & Al realizar un examen me siento motivado a seguir aprendiendo & & & & \\
\hline 8 & Cuando mi valoración definitiva es inferior a siete, siento que no he aprendido y comprendido lo trabajado. & & & & \\
\hline 9 & Cuando mi valoración definitiva es superior a siete, siento que he comprendido lo trabajado. & & & & \\
\hline 10 & Una buena nota me representa haber comprendido un tema. & & & & \\
\hline 11 & Me siento bien cuando las evaluaciones son escritas & & & & \\
\hline 12 & Me siento bien cuando las evaluaciones son orales. & & & & \\
\hline 13 & La evaluación es un proceso gestionado por el docente, con mi participación. & & & & \\
\hline 14 & $\begin{array}{l}\text { Autoevaluó mi proceso de aprendizaje y doy valoración a mi trabajo, para tomar decisiones de cambio con respecto } \\
\text { a mi formación escolar. }\end{array}$ & & & & \\
\hline 15 & Cuando recibo los resultados de una evaluación conozco los criterios con los cuales he sido evaluado & & & & \\
\hline 16 & Se tienen en cuenta mis opiniones acerca de mi proceso de aprendizaje cuando se me evalúa & & & & \\
\hline 17 & $\begin{array}{l}\text { En el proceso de evaluación así como el profesor evalúa a sus estudiantes, los estudiantes evalúan la gestión de su } \\
\text { profesor. }\end{array}$ & & & & \\
\hline 18 & Al iniciar un tema se realiza una sensibilización en la que se identifican los conocimientos previos. & & & & \\
\hline 19 & La evaluación me sirve para identificar mis errores conceptuales y no repetirlos. & & & & \\
\hline 20 & Generalmente estudio y me preparo para una evaluación. & & & & \\
\hline 21 & La evaluación es una forma de cuantificar mis conocimientos & & & & \\
\hline 22 & Mis compañeros evalúan mi proceso de aprendizaje y de la misma forma yo los evaluó a ellos, para mejorar. & & & & \\
\hline 23 & Las preguntas del examen se relacionan con las temáticas revisadas en clase. & & & & \\
\hline 24 & En los exámenes me preguntan situaciones problema que no tienen relación con lo visto en clase & & & & \\
\hline 25 & Lo que se evalúa es importante en mi proceso de aprendizaje. & & & & \\
\hline 26 & Prestar atención en clase permite un buen resultado en mi evaluación. & & & & \\
\hline 27 & En el examen me preguntan diversos aspectos observados en diversos talleres experimentales. & & & & \\
\hline 28 & El profesor discute y acuerda la valoración o porcentajes que atribuirá a cada nota con los estudiantes. & & & & \\
\hline
\end{tabular}

29. Sugiere ideas de cómo te gustaría que evaluaran tu proceso de aprendizaje:

30. Resalte aspectos positivos que en la clase de química se tienen en cuenta dentro del proceso de evaluación individual y grupal.

Cordialmente: Adny Lorena Vásquez, Julieth Katherine Orobio Profesoras en formación Laksmi Latorre, profesora titular. Sandra Ximena Ibáñez. Asesora de Práctica Pedagógica y Didáctica-UPN 